\title{
Holographic Pomeron and the Schwinger Mechanism
}

\section{Gökçe Başar*}

Department of Physics and Astronomy, Stony Brook University, Stony Brook, New York 11794-3800, USA

E-mail: basaretonic.physiscs. sunysb.edu

\section{Dmitri E. Kharzeev}

Department of Physics and Astronomy, Stony Brook University, Stony Brook, New York 11794-3800, USA

Department of Physics, Brookhaven National Laboratory, Upton, New York 11973-5000, USA

E-mail: dmitri.kharzeevestonybrook.edu

\section{Ho-Ung Yee}

Department of Physics and Astronomy, Stony Brook University, Stony Brook, New York 11794-3800, USA

E-mail: hyee@tonic.physiscs.sunysb.edu

\section{Ismail Zahed}

Department of Physics and Astronomy, Stony Brook University, Stony Brook, New York 11794-3800, USA

E-mail: zahedetonic.physiscs.sunysb.edu

I revisit the problem of dipole-dipole scattering via exchanges of soft Pomerons in the context of holographic QCD with confinement and show that a single closed string exchange contribution to the eikonalized dipole-dipole scattering amplitude yields a Regge behavior of the elastic amplitude. I provide a physical interpretation of the semi-classical worldsheets driving the Regge behavior for $(-t)>0$ in terms of worldsheet instantons which describe the Schwinger mechanism for string pair creation by an effective electric field induced by the relative rapidity $\chi$ of the scattering dipoles. The analysis naturally explains the diffusion in the impact parameter space encoded in the Pomeron exchange via the Unruh temperature of accelerated strings under the electric field. I also argue for the existence of a "micro-fireball" in the middle of the transverse space due to the soft Pomeron exchange, which may be at the origin of the thermal character of multiparticle production in ep/pp collisions. After summing over uncorrelated multi-Pomeron exchanges, I show that the total dipole-dipole cross section obeys the Froissart unitarity bound.

Sixth International Conference on Quarks and Nuclear Physics,

April 16-20, 2012

Ecole Polytechnique, Palaiseau, Paris

${ }^{*}$ Speaker. 


\section{Introduction}

Non-perturbative aspects of near-forward, high energy hadronic scattering is a longstanding problem and carry valuable information about the total cross section and particle multiplicities. Even though the perturbative description of the leading t-channel exchange (the Pomeron) as Reggeized gluons [1] is qualitatively consistent with the observed growth of the scattering amplitude [2], some empirical features of the hadron-hadron scattering (e.g. the Pomeron slope) point to the importance of non-perturbative effects.

In this talk, I will consider gauge-string duality (or holography) to issue this problem. The use of holography in high energy scattering and Pomeron physics has been addressed several times in the past. In [3], exchanges of Minkowskian string surfaces in $A d S_{5}$ has been considered. A similar semi-classical was pursued in [4] to address the same problem in the confining background of [5]. A different perspective to the problem has been taken in [6]. In this talk, I will explain the approach advocated in [7] which shares a similar philosophy with [4].

In particular, I consider elastic dipole-dipole scattering $D_{1}\left(p_{1}\right)+D_{2}\left(p_{2}\right) \rightarrow D_{1}\left(k_{1}\right)+D_{2}\left(k_{2}\right)$ with a dipole size $a$. The kinematics is given by $s=\left(p_{1}+p_{2}\right)^{2}, t=\left(p_{1}-k_{1}\right)^{2}, s+t+u=4 m^{2}, p_{1} / m=$ $\left(\cos (\theta / 2),-\sin (\theta / 2), 0_{\perp}\right), p_{2} / m=\left(\cos (\theta / 2), \sin (\theta / 2), 0_{\perp}\right), q=\left(0,0, q_{\perp}\right)$ and $b=\left(0,0, b_{\perp}\right)$, where $q$ is the t-channel momentum $\left(t=-q_{\perp}^{2}<0\right)$ and $b$ is the impact parameter. In Euclidean signature, the relative rapidity $\chi$ translates into a Euclidean angle $\theta$ with $\cosh \chi=\frac{s}{2 m^{2}}-1 \rightarrow \cos \theta$. Scattering at high-energy in Minkowski geometry follows from analytically continuing $\theta \rightarrow-i \chi$ in the regime $\chi \approx \log \left(s / 2 m^{2}\right) \gg 1[8]$.

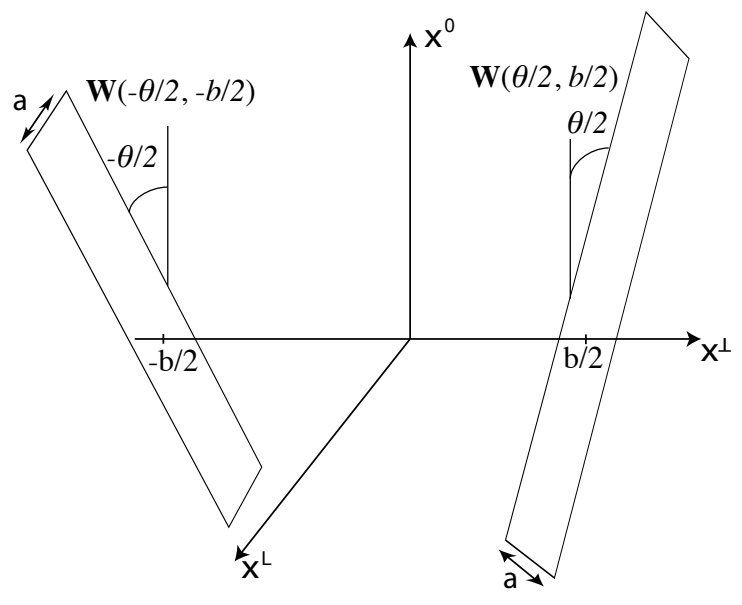

Figure 1: Dipole-dipole scattering configuration in Euclidean space. The dipoles have size $a$ and are $b$ apart. The dipoles are sloped by an angle $\pm \theta / 2$ (Euclidean rapidity) in the longitudinal $x^{0} x^{L}$ plane.

Using the eikonal approximation, LSZ reduction and the analytic continuation discussed above, the dipole-dipole scattering amplitude $\mathscr{T}$ can be reduced to a correlation function of Wilson loops and in Euclidean space takes the following form [9]

$$
\frac{1}{-2 i s} \mathscr{T}(\theta, q) \approx \int d^{2} b e^{i q_{\perp} \cdot b}\langle\mathbf{W}(-\theta / 2,-b / 2) \mathbf{W}(\theta / 2, b / 2)-\mathbf{1}\rangle
$$

The key ingredient for the scattering amplitude is the dipole-dipole correlation function $<\mathbf{W W}\rangle$. 


\section{Holographic Calculation of the Scattering Amplitude}

In holography, the dipole-dipole correlator $<\mathbf{W W}>$ is identified with the partition function of the dual string propagating in the higher dimensional confining background $[5,10]$. The string worldsheet is bounded by the Wilson loops in Fig 1 which sit on the UV boundary of the holographic geometry. For small dipoles and large impact parameter $b$, most of the string worldsheet stays at the IR end point of the confining geometry and propagates in the flat space-time with an effective string tension. It is shown in [7] that the contribution of the fermionic sector is suppressed for the particular kinematical regime $\chi>>\lambda$ where $\lambda>>1$ is the 't Hooft coupling. As a result, the Pomeron amplitude at large rapidity, translates into the bosonic cylinder partition function in the string side. The cylinder topology is necessary since it is the topology of the Pomeron in $1 / N_{c}$ expansion.

$$
<\mathbf{W W}>=g_{s}^{2} \int_{0}^{\infty} \frac{d T}{2 T} \int_{\mathrm{T}} d[x] e^{-\mathbf{S}[x]+\text { ghosts }}=g_{s}^{2} \int_{0}^{\infty} \frac{d T}{2 T} \mathbf{K}(T)
$$

where $\mathbf{S}$ is the standard Polyakov action,

$$
\mathbf{S}=\frac{\sigma_{T}}{2} \int_{0}^{T} d \tau \int_{-b / 2}^{b / 2} d \sigma\left(\dot{x}^{\mu} \dot{x}_{\mu}+x^{\prime \mu} x_{\mu}^{\prime}\right)
$$

subject to sloped boundary conditions of the dipole configuration shown in Fig. 1:

$$
x^{\mu}(T, \sigma)=x^{\mu}(0, \sigma) \quad, \quad \cos (\theta / 2) x^{L}(\tau, \mp b / 2) \pm \sin (\theta / 2) x^{0}(\tau, \mp b / 2)=0
$$

The parameter $T$ in (2.1) is the modulus of the cylinder, analogous to proper time. Here I have assumed that the dipole size $a$ is much smaller than the impact parameter $b$. In this limit, the rectangular Wilson loops on the boundaries simply shrink into lines. The path integral in (2.1) with the sloped boundary conditions (2.3) can be evaluated in a straightforward fashion by mode expansion and zeta function regularization [7] and leads to the propagator

$$
\mathbf{K}(T)=\pi \sigma_{T} a^{2} e^{-\sigma_{T} b^{2} T / 2} \eta^{-D_{\perp}}(i T / 2) \frac{1}{\sinh (\theta T / 2)} \prod_{n=1}^{\infty} \prod_{s= \pm} \frac{\sinh (\pi n T / 2)}{\sinh (\pi(n+s \theta / \pi) T / 2)}
$$

In this expression, the term inside the exponent is the classical world-sheet area. The next term, $\eta(i T / 2)$, is the Dedekind eta function, and comes from the transverse fluctuations. $D_{\perp}$ is the number of transverse dimensions. The $\sinh ^{-1}$ is the contribution of the longitudinal zero mode, and the infinite product is the contribution of the ghosts and non zero longitudinal modes.

After analytical continuation $\theta \rightarrow-i \chi$ to Minkowski space, the $\sinh ^{-1}$ term develops poles on the real axis for $T=2 k \pi / \chi$. Deforming to contour below the negative poles and above the positive poles results in picking only the positive poles whose residues are purely real:

$$
<\mathbf{W W}>_{\text {poles }}=\frac{g_{s}^{2} a^{2}}{4 \alpha^{\prime}} \sum_{k=1}^{\infty} \frac{(-1)^{k}}{k} e^{-k b^{2} / 2 \chi \alpha^{\prime}} \eta^{-D_{\perp}}(i k \pi / \chi)
$$

The expression (2.5) corresponds to the production rate of a " $k$-string" with tension $k \sigma_{T}$ (see [7] for details). However since the boundaries are Wilson lines, not D0 branes, the strings with $k>1$ 
need to have multiple wrappings and therefore their contributions are further suppressed by factors of $1 / N_{c}^{2}$. The leading term is the $k=1$ pole which gives

$$
<\mathbf{W W}>=-a^{2} \pi g_{S}^{2}\left(4 \pi \alpha^{\prime}\right)^{D_{\perp}-1}\left(\frac{1}{2 \pi \alpha^{\prime} \chi}\right)^{D_{\perp} / 2} e^{-b^{2} / 2 \alpha^{\prime} \chi+D_{\perp} \chi / 12}
$$

at large rapidity. For notational simplicity, I will denote this leading pole contribution as $\langle\mathbf{W W}\rangle$ from now on. From (1.1) the scattering amplitude is found as

$$
\mathscr{T}(s, t)=-2 i s \int d^{2} b e^{i q_{\perp} b}<(\mathbf{W W}-1)>=i a^{2} \pi^{2} g_{s}^{2}\left(\frac{\pi}{\ln s}\right)^{D_{\perp} / 2-1} s^{\alpha(t)} .
$$

Note that, the elastic amplitude (2.7) is purely imaginary, not a pure phase, signaling the inelasticity. This inelasticity is tied to the exchange of strings. The associated Regge trajectory is given by $\alpha(t)=1+\frac{D_{\perp}}{12}+\frac{\alpha^{\prime}}{2} t$. The slope of the trajectory is given by the half of $\alpha^{\prime}$, consistent with the topology of two gluon exchange. In contrast with [6], the intercept $1+D_{\perp} / 12$ is in general different from 2, which is simply the spin of the graviton. This is expected because we have a non-critical string with no fermionic degrees of freedom thus no massless graviton exist in the spectrum. In fact, the term $D_{\perp} / 12$ is the Casimir energy or the Lüscher correction [11] of the bosonic fluctuations around the classical string world-sheet, as also pointed out (although with a different value) in [4]. Also recalling that the supersymmeries of the gauge theory are associated with isometries extra dimensions in the dual geometry, it is conceivable to assume $D_{\perp}=2$ to account for a non supersymmetric gauge configuration. Indeed, in Sakai-Sugimoto model, the kinematical limit $\chi>>\lambda$ is identical with the limit where the impact parameter is much larger than the curvature scale of the extra dimensions [7], and the fluctuations in these directions should be suppressed. With $D_{\perp}=2$, the intercept is

$$
\alpha(0) \approx 1.17 \quad\left(D_{\perp}=2\right)
$$

This result is in between the BFKL [1] or "hard pomeron" value $(\alpha(0) \approx 1.3)$ and the experimental fit of Donnachie and Landshoff $(\alpha(0) \approx 1.08)$ [12]. The discrepancy might be due to the fact that the evolution in dipole sizes and multiple pomeron interactions have not been taken into account in this analysis. The former point is further pursued in [13]. Finally, the total cross section is given by the forward $(t=0)$ amplitude

$$
\sigma_{t o t}=a^{2} \pi^{2} g_{s}^{2}\left(\frac{\pi}{\ln s}\right)^{D_{\perp} / 2-1} s^{D_{\perp} / 12}=a^{2} \pi^{2} g_{s}^{2} s^{0.17} \quad\left(D_{\perp}=2\right)
$$

\section{Gribov Diffusion and Schwinger Mechanism}

The Regge behavior of the scattering amplitude naturally describes a diffusion in the transverse space. These two are simply related by a Fourier transform. Factoring out the normalized diffusion kernel from the expression (2.7)

$$
\mathbf{K}(\chi, b)=\left(\frac{1}{2 \pi \alpha^{\prime} \chi}\right)^{D_{\perp} / 2} e^{-b^{2} / 2 \chi \alpha^{\prime}}
$$


shows that the dipole-dipole correlator satisfies the diffusion equation $\partial_{\chi} \mathbf{K}(\chi, b)=\alpha^{\prime} / 2 \nabla_{\perp}^{2} \mathbf{K}(\chi, b)$. This diffusion phenomenon was explained by Gribov [14] in the context of random walk in the parton evolution. An alternative perspective for this phenomenon can be constructed in terms of the Schwinger mechanism for string production in the presence of an effective electric field driven by the rapidity $\chi$. A world-sheet T-duality along the longitudinal direction $x^{L} \rightarrow \tilde{x}^{L}$ is useful for this purpose. The T-duality acts as: $\partial_{\tau} x^{L}=\partial_{\sigma} \tilde{x}^{L}, \partial_{\sigma} x^{L}=\partial_{\tau} \tilde{x}^{L}$ and converts the sloped boundary condition (2.3) into an effective electric field $E=\sigma_{T} \tanh (\chi / 2)$ acting on the two end points of the open strings stretching between the two dipoles. The Polyakov action then becomes

$$
S=\frac{\sigma_{T}}{2} \int_{0}^{T} d \tau \int_{-b / 2}^{b / 2} d \sigma\left(-\left(\partial x^{0}\right)^{2}+\left(\partial \tilde{x}^{L}\right)^{2}+\left(\partial x^{\perp}\right)^{2}\right)+\left.\frac{E}{2} \int d \tau\left(\tilde{x}^{L} \partial_{\tau} x^{0}-x^{0} \partial_{\tau} \tilde{x}^{L}\right)\right|_{\sigma= \pm b / 2}
$$

The signs of the electric fields on both ends are opposite due to the opposite direction of motions of the two dipoles, but the two end points of a string carry opposite charges, so that there is a net acceleration. The dominant contribution of (3.2) to the string partition function comes from saddle points in proper time $T$

$$
\begin{array}{lll}
x^{0}=R(\sigma) \cos (2 \pi k / T \tau), & \tilde{x}^{L}=R(\sigma) \sin (2 \pi k / T \tau), & x^{\perp}=\sigma \\
R(\sigma)=b / \chi \cosh (\chi / b \sigma) & , \quad T=2 \pi k / \chi \quad, \quad k \in \mathbb{Z}^{+}
\end{array}
$$

These configurations are also known as world-sheet instantons [15]. The saddle points occur at $T=$ $2 \pi k / \chi$ which coincide with poles of (2.4). For a given positive integer $k$, each instanton trajectory (3.3) describes a string wrapping $k$ times around a circle in Euclidean $x^{0} \tilde{x}^{L}$ plane. The classical action evaluated on the trajectory is $S_{c l}=k \frac{\pi b^{2} \sigma_{T}}{\chi}$. Holography then identifies the string production rate $e^{-S_{c l}}$ with the scattering amplitude. As a result, it is ultimately the world-sheet instantons which seed the diffusion in transverse space and Regge behavior of the scattering amplitude. The fluctuations around the classical solution (3.3) or the "instanton determinant" contributes both as the normalization of the diffusion kernel and as the additional term $D_{\perp} / 12$ to the intercept.

Furthermore, the Euclidean circular motion in (3.3) becomes acceleration in Minkowski space. The radius of the circle, $R(\sigma)$, is smallest in the center of the string and increases exponentially towards the dipoles. In Minkowski space, the inverse radius is the acceleration. The accelerated string feels an Unruh temperature given by $T(\sigma)=2 \pi / R(\sigma)$. At the center, the temperature is $T(0)=\chi / 2 \pi b$ and it decreases exponentially as one moves away from the center. This picture relates the Gribov diffusion with the thermal nature of the string production via the Unruh effect. Remarkably, for $\chi>>\lambda$, the Unruh temperature $T(0)$ at the center is greater than the deconfinement temperature in Sakai-Sugimoto model. As a result, the string exchange at large rapidity creates to a small region of deconfined matter in the middle of the collision. The existence of this "micro fireball" from a single soft Pomeron exchange can naturally explain the observed apparent thermal nature of multiparticle production in high energy collisions and might be the seed of the quark gluon plasma observed in AA collisions observed in RHIC and LHC.

\section{Froissart Bound}

The scattering amplitude (2.7) violates unitarity at large rapidity. This is due to the fact that the 
dipole-dipole correlator describes only a single pomeron exchange and this approximation can break down at large rapidity. One conventional way to overcome this problem is to sum over the non-interacting multi pomeron exchanges which corresponds to exponentiating the single pomeron correlator:

$$
\frac{1}{2 i s} \mathscr{T}(s, t)=\int d^{2} b e^{i q \cdot b}(1-\exp (<\mathbf{W W}>)) .
$$

Through the optical theorem, the total cross section is found to be

$$
\sigma_{t o t}=2 \int d^{2} b\left(1-e^{<\mathbf{W W}>}\right)
$$

For large rapidity, the integrand in (4.2) vanishes for $b>b_{\max }=\sqrt{D_{\perp} \frac{\alpha^{\prime}}{6}} \chi$ since $<\mathbf{W W}>\sim$ $\exp \left(D_{\perp} \chi / 12-b^{2} / 2 \alpha^{\prime} \chi\right) \rightarrow 0$. Furthermore it is equal to unity for $b \leq b_{\max }$ as the second term in the integrand is exponentially small. This is the well known "black disk" limit where the cross section has a sharp transition between 1 and 0 at $b=b_{\max }$. The total cross section at large rapidity is

$$
\sigma_{\mathrm{tot}}(s) \approx 2 \int^{b_{\max }} d^{2} b=\frac{\pi D_{\perp} \alpha^{\prime}}{3} \chi^{2}
$$

As a result, the total cross section (4.3) saturates the Froissart unitarity bound, i.e. $\sigma_{\mathrm{tot}}(s) \leq \chi^{2}$ [16]. The dimension of the total cross section is naturally given by the string tension.

\section{References}

[1] E. A. Kuraev, L. N. Lipatov and V. S. Fadin, Sov. Phys. JETP 44, 443 (1976), L. N. Lipatov, Sov. J. Nucl. Phys. 23, 338 (1976).

[2] S. Eidelman et al. [Particle Data Group Collaboration], Phys. Lett. B 592, 1 (2004).

[3] M. Rho, S. -J. Sin, I. Zahed, Phys. Lett. B466, 199-205 (1999).

[4] R. A. Janik, R. B. Peschanski, Nucl. Phys. B586, 163-182 (2000), Nucl. Phys. B625, 279-294 (2002),

R. A. Janik, Phys. Lett. B 500, 118 (2001), M. Giordano and R. Peschanski, JHEP 1110, 108 (2011).

[5] E. Witten, Adv. Theor. Math. Phys. 2, 505 (1998),

[6] R. C. Brower, J. Polchinski, M. J. Strassler and C. I. Tan, JHEP 0712, 005 (2007)

[7] G. Basar, D. E. Kharzeev, H. -U. Yee and I. Zahed, Phys. Rev. D 85, 105005 (2012)

[8] E. Meggiolaro, Z. Phys. C 76, 523 (1997), Eur. Phys. J. C 4, 101 (1998), Nucl. Phys. B 625, 312 (2002), M. Giordano and E. Meggiolaro, Phys. Lett. B 675, 123 (2009).

[9] O. Nachtmann, Ann. Phys. 209 (1991) 436;

[10] T. Sakai and S. Sugimoto, Prog. Theor. Phys. 113, 843 (2005).

[11] M. Lüscher, Nucl. Phys. B 180, 317 (1981).

[12] A. Donnachie and P. V. Landshoff, Nucl. Phys. B 244, 322 (1984).

[13] A. Stoffers and I. Zahed, arXiv:1205.3223 [hep-ph].

[14] V. N. Gribov, hep-ph/0006158.

[15] C. Schubert, J. Phys. Conf. Ser. 287, 012031 (2011)

[16] M. Froissart, Phys. Rev. 123, 1053 (1961). 